\title{
DEMOGRAPHIC AND DRIVING PERFORMANCE FACTORS IN SIMULATOR ADAPTATION SYNDROME
}

\author{
Matthew Rizzo, Rebecca A. Sheffield, \\ Laura Stierman, Jeffrey Dawson \\ Colleges of Medicine, Engineering, and Public Health \\ Public Policy Center \\ University of Iowa \\ Iowa City, IA, USA
}

\begin{abstract}
Summary: Simulation is an important option for testing at-risk drivers with medical impairments. Simulator Adaptation Syndrome (SAS), characterized by autonomic symptoms, presents a drawback to testing. This study investigated new issues regarding susceptibility of neurologically impaired drivers to SAS, scenario situations most likely to cause SAS, and effects of SAS on driver performance. Subjects were 164 drivers enrolled in larger ongoing studies of at-risk older drivers. Eighteen had Alzheimer's disease (AD), 44 stroke, and 102 were neurologically normal controls. Experimental drives were conducted using a fixed-base high-fidelity simulator with a $150^{\circ}$ forward field of view. Each driver completed a questionnaire immediately after driving in the simulator, rating any feelings of discomfort along nine dimensions; an overall discomfort score was calculated. Of the 164 drivers, 130 completed the full drive and 34 ended the drive early. Drivers with higher overall discomfort scores were more likely to drop out before completing a drive. Specific symptoms strongly predicted dropping out, namely dizziness, nervousness, light-headedness, body temperature increase, and nausea. Simulator dropout rates and reported discomfort scores were significantly greater in women than men, but did not differ between drivers with $\mathrm{AD}$ or stroke and neurologically normal drivers. Comparisons between 32 Dropouts and 32 Non-Dropouts (matched by age, gender, neurological impairment, and scenario driven) showed no evidence that higher levels of discomfort cause a driver to perform atypically before the point of dropout. We could relate dropout to specific segments and events in the drive that required abrupt braking.
\end{abstract}

\section{INTRODUCTION}

As simulation becomes a more popular tool for driving safety research, adaptation and acceptance of virtual environments by human subjects immersed in simulation tasks are becoming key issues. This is especially true of simulator adaptation syndrome (SAS). SAS is characterized by a range of complaints, most notably autonomic symptoms including nausea, and presents a drawback to simulation research. Discomfort may be due to a mismatch between visual cues of movement, which are plentiful, and inertial cues, which are lacking or imperfect, even in simulators with a motion base. This resembles motion sickness, in which a person enclosed in an elevator or below deck in a boat is exposed to strong inertial cues in the absence of matching visual cues (Rizzo, 2003). 
Stanney et al. (1998) calculated that $80 \%$ to $95 \%$ of participants across studies in a number of virtual environments reported adverse symptoms, and 5\% to $30 \%$ experienced symptoms severe enough to end participation. The wide variation in the incidence and severity of symptoms probably reflects exposure to a broad range of simulator displays, devices, technologies, and scenarios. A committee that considered these symptoms emphasized the need for (a) standard measurement approaches and (b) identification of sensorimotor discordances that underlie these adverse effects (Stanney and Salvendy, 1998).

Previous research on simulator adaptation has focused on gender. Women have reported higher symptoms of simulation discomfort than men on post-drive questionnaires (Kennedy et al., 1995). However, few data exist on features such as medical impairment, aging, and drivingrelated performance factors. Stanney and Hash (1998) suggested that user-initiated control affects adverse symptoms. Along these lines, does poor control of a simulator vehicle trigger driver SAS or does SAS impair simulated vehicle control?

This study investigates new issues regarding susceptibility of neurologically impaired drivers to SAS, scenario situations most likely to trigger SAS, and effects of SAS on driver performance. Hypothesized factors in SAS, besides advanced age and female gender, are neurological disease, and excessive braking and steering. We assessed these demographic and driver performance factors in SAS using a fixed-base high-fidelity simulator, as outlined below.

\section{METHODS}

Subjects:164 drivers (53.1\% men) participated in drives on a fixed-base high-fidelity driving simulator. Of these drivers, 102 had no known neurological disorder, 44 had stroke, and 18 had Alzheimer's disease. All participants were tested with a battery of visual and neuropsychological tests as part of ongoing studies of cognition and driving performance. Exclusion criteria included a history of motion sickness, vestibular disease, active depression, substance abuse, and acute medical illness.

Driving Simulator: The Simulator for Interdisciplinary Research in Ergonomics and Neuroscience (SIREN) is a fixed-base driving simulator designed to create an immersive realtime virtual environment for assessing at-risk drivers in a medical setting in the University of Iowa Hospitals. SIREN comprises a complete four-door 1994 GM Saturn SL2 cab, with specially embedded electronic sensors and infrared illuminated pinhole video cameras for recording driver performance. It includes a sound system, surrounding screens $\left(150^{\circ}\right.$ forward $\mathrm{FOV}, 50^{\circ}$ rear FOV), four LCD projectors with image generators, an integrated host computer, and a desktop computer for scenario design, control, and data collection. A tile-based scenario development tool (DriveSafety, Fort Collins) allows us to generate multiple road types and vehicles that interact with the driver and each other.

Each subject drove on a simulated rural 2-lane highway with interactive traffic resembling a drive on the roads surrounding Iowa City, IA. The simulation consists of several "events" associated with potential crashes interspersed with uneventful highway segments. Depending on speed, the simulated drives lasted up to 30 minutes. 
Driving measures: Dependent measures of driver control over SIREN included steering and braking control. Steering control was indexed by the standard deviation of the steering wheel position throughout the drive. The rate of the steering wheel reversals of at least 6 degrees in either direction was also calculated to measure erratic steering (Dingus et al., 1989). Abrupt braking was indexed by number of brake pedal depressions of $30 \%$ or more of full possible pedal excursion.

Discomfort assessment: A Simulator Adaptation Questionnaire (SAQ) rated the visual, perceptual, and physiological experience of driving in SIREN. We developed this brief tool in consideration of items from the Simulator Sickness Questionnaire (SSQ) of Kennedy et al. (1993) and Motion Sickness Questionnaire (MSQ) of Kellogg et al. (1965), and our own observations and experience with subject reports after driving simulation. We tried to avoid redundancy and excluded items that had not arisen in our previous studies (e.g., "desire to move the bowels" from the MSQ). The SAQ rates sensations of discomfort along nine dimensions (body temperature increase, boredom, dizziness, eye strain, headache, light-headedness, nausea, nervousness, sleepiness $)$ on a scale from 1 to $7(1=$ no discomfort; $7=$ extreme discomfort $)$. These individual scores were combined to assess total discomfort (range 9 to 63).

\section{RESULTS}

Of the 164 subjects, 130 completed the full drive in SIREN and 34 (Dropouts) ended the drive early (Table 1).

Table 1. Demographic factors of drivers

\begin{tabular}{|l|l|l|l|}
\hline & $\begin{array}{l}\text { All subjects } \\
(\mathbf{n = 1 6 4 )}\end{array}$ & $\begin{array}{l}\text { Dropouts } \\
(\mathbf{n = 3 4 )}\end{array}$ & $\begin{array}{l}\text { Non-Dropouts } \\
(\mathbf{n = 1 3 0})\end{array}$ \\
\hline Age (SD) years, age range & $\begin{array}{l}67.8(9.68), \\
35 \text { to } 89\end{array}$ & $\begin{array}{l}67.9(10.4), \\
47 \text { to } 86\end{array}$ & $\begin{array}{l}67.8(9.54), \\
35 \text { to } 89\end{array}$ \\
\hline Gender (\% Men) & $53.1 \%(87 \mathrm{M}, 77 \mathrm{~F})$ & $26.5 \%(9 \mathrm{M}, 25 \mathrm{~F})$ & $60.0 \%(78 \mathrm{M}, 52 \mathrm{~F})$ \\
\hline AD & 18 & 5 & 13 \\
\hline Stroke & 44 & 11 & 33 \\
\hline Neurologically Normal & 102 & 18 & 84 \\
\hline
\end{tabular}

Thirty-two of the 34 Dropouts could be matched to 32 Non-Dropouts, on criteria of gender, age, neurological impairment, and scenario driven. Each Non-Dropout driver's file was truncated to match the duration of the corresponding Dropout's file. Data from the 32 Dropouts and 32 matched Non-Dropouts were analyzed to test whether discomfort scores and driving measures were related to dropout status. 
Table 2. Demographic Characteristics Dropouts and Non-Dropouts

\begin{tabular}{|l|l|l|}
\hline & Dropouts $(\mathbf{n}=32)$ & Completers $(\mathbf{n}=32)$ \\
\hline Age (SD) years, age range & $\begin{array}{l}68.3(9.99), \\
47 \text { to } 86\end{array}$ & $\begin{array}{l}67.3(9.42), \\
44 \text { to } 83\end{array}$ \\
\hline Gender (\% Men) & $28.1 \%(9 \mathrm{M}, 23 \mathrm{~F})$ & $28.1 \%(9 \mathrm{M}, 23 \mathrm{~F})$ \\
\hline AD & 4 & 4 \\
\hline Stroke & 10 & 10 \\
\hline Neurologically Normal & 18 & 18 \\
\hline
\end{tabular}

\section{HYPOTHESES}

1. Discomfort scores and dropout rate increase with advancing age, female gender, and neurologic impairment.

We used Wilcoxon Rank Sum tests and Spearman correlations to assess if discomfort scores were related to age, gender, and/or impairment status, and Chi-square tests of independence to assess whether these variables were related to dropping out. Contrary to expectation, younger subjects tended to report higher discomfort scores $(\mathrm{r}=-0.178, \mathrm{P}=0.028)$, but age was not related to whether subjects dropped out. Women reported higher discomfort scores $(\mathrm{P}=0.015)$ and were more likely to end the drive early than men $(\mathrm{P}<0.001)$. Simple analyses indicated neurological impairment had no effect on discomfort score or dropout rate. Controlling for age and gender using logistic regression, impaired subjects (AD and stroke together) did not predict discomfort scores, however the impaired were more likely to drop out $(\mathrm{OR}=2.412,95 \% \mathrm{CI}:(1.014,5.741)$.

2. Discomfort scores and dropout rate increase with greater steering variability and greater frequency of abrupt braking and abrupt steering maneuvers.

Steering and braking measures were not related to the discomfort score, but the subjects who dropped out did brake more frequently than those who did not (Wilcoxon Rank Sum $\mathrm{P}=0.041$ ). Fifteen of the 34 Dropouts stopped the drive after the Go/No-Go section, which requires increased braking.

3. Drivers who drop out have higher discomfort scores than those who do not.

Using Wilcoxon Rank Sum tests, subjects who dropped out reported significantly higher total discomfort $(\mathrm{P}=0.021)$ as well as nervousness $(\mathrm{P}=0.005)$, dizziness $(\mathrm{P}=0.004)$, light headedness $(\mathrm{P}=0.011)$, body temperature increase $(\mathrm{P}=0.025)$, and nausea $(\mathrm{P}=0.025)$.

\section{DISCUSSION}

Driving in a simulator produces a range of experiences among drivers. Using safety protocols under which subjects could curtail research participation at any time, almost $80 \%$ of 164 drivers completed a full drive. No driver became "sick" (i.e., none vomited), militating against the 
daunting terms, "simulator sickness" or "cybersickness." The broad range of symptoms we observed is probably better subsumed under by a more neutral term such as "simulator adaptation syndrome."

Symptoms: In this study, drivers with higher overall discomfort scores in the SAQ were more likely to drop out before completing a drive, in line with Hypothesis 3. Specific symptoms strongly predicted dropping out, namely dizziness, nervousness, light-headedness, body temperature increase, and nausea. Dizziness and lightheadedness are colloquial terms often used by patients reporting to vestibular disorders clinics (Fattal and Rizzo, 2003). Reports of body temperature increase and nausea are broadly compatible with autonomic symptoms (as may occur with vestibular complaints). Nervousness is a non-specific term that may accompany vestibular or autonomic symptoms, and is often used to describe psychological symptoms that are not simulation-related (e.g., performance anxiety on a challenging task). Symptoms of boredom, eye strain, headache, or sleepiness also did not discriminate between Dropouts and Non-Dropouts and might be eliminated from shorter questionnaire tools.

Demographic Factors: This study tested demographic risk factors for SAS and dropout related to age, gender and neurologic disease. The women in our sample of 164 drivers reported significantly higher discomfort scores and were more likely to drop out than the men as Hypothesis 1 and reviewed literature predicted. Taken at face value, these results would reflect true differences in how men and women experience simulation, although there may be alternative explanations (see below).

Contrary to prediction, older drivers did not report greater discomfort than younger drivers. Also, neither the AD group nor the stroke group generated higher SAQ scores or were more likely to drop out than the neurologically normal drivers. After we controlled for age and gender, impaired subjects (AD and stroke together) still did not have significantly higher discomfort scores, although the combined groups were now more likely to drop out.

Driver dropout in the absence of increased symptom scores, measured using self-report tools such as the SAQ, could be due to a variety of reasons. Some drivers may have failed to complete a drive for reasons other than SAS. For example, some might have been discouraged by their perceived performance, fatigued, or under pressure of time (e.g., to return home). In a different vein, some drivers might underreport their discomfort and instead disclose (a) what they think they should have felt or the examiners want to hear, or (b) what they want the examiners to hear. For instance, a neurologically impaired driver might hide discomfort and try harder to complete a drive despite discomfort to avoid appearing "weak." Similarly, men might feel compelled to complete a drive and report less discomfort because reporting illness symptoms to others may be more socially acceptable for women (Hibbard and Pope, 1983; Thompson and Brown, 1980). Women may be more susceptible to discomfort when historical or questionnaire data are used, but not when physiological measures are used (Park and Hu, 1999).

Synchronous recording of physiologic measures such as Galvanic skin response, heart rate and respiration being implemented in our ongoing simulator studies (Rizzo et al., 2003), can help reveal links or mismatches between symptom reports and physiology in different populations of drivers. Application of such tools in drivers of instrumented vehicles could help ascertain the extent to which automobile driving itself contributes to symptoms, outside of simulation. 
Under human subject protections, this study excluded drivers with a self-reported history of motion sickness, which may affect up to $10 \%$ of the general population (Cass et al., 1997). We had to assume these drivers would be overly prone to simulator discomfort, although this remains unproven. Migraine headache is another possible risk factor for SAS (Viirre and Bush, 2002). Migraine affects 17-29\% of females and 6-20\% of males (Cass et al., 1997) and up to $25 \%$ of these patients report dizziness (Baloh, 1997). Headache was not an exclusion criterion in this study, however headache symptoms on the SAQ did not discriminate between Dropouts and Non-Dropouts in this study. Future studies can more directly address the role of migraine headache in SAS.

Driver Performance: This study also evaluated performance factors related to SAS. Hypothesis 2 asked if specific driver behaviors like abrupt or excessive braking and steering in drivers who failed to master control over the simulator, or certain simulator scenarios that may require abrupt maneuvers (by any driver), might provoke discomfort or dropout. We also asked whether driving performance declined due to increased discomfort. For example, drivers with poor steering control might be more susceptible to discomfort or drop out from the to-and-fro sweep of panoramic cues (i.e., motion vection cues) across their peripheral visual fields. However, our results showed that steering measures were not related to discomfort scores or dropout. Likewise, braking measures were not related to discomfort scores. Drivers who dropped out did brake more frequently than those who did not $(\mathrm{P}=0.041)$, especially in scenarios that might require abrupt braking, such as Go/No-Go scenarios. These instances could be clearly identified in the record and, if needed, considered separately from other driving performance data.

In short, we found no evidence that higher levels of discomfort cause a driver to perform atypically before the point of drop out. In other words, simulation data do not appear to underestimate performance of drivers with higher SAS scores or those who drop out before completing a drive.

\section{CONCLUSION}

Use of a simulator provides key options for safely testing drivers in potentially unsafe situations such as collision avoidance or distraction and for testing "at-risk" drivers with medical impairments. To optimize these simulator applications we must better understand demographic risk factors, and physiologic and behavioral correlates of SAS. This includes a better understanding of visual-vestibular mechanisms that SAS may share with "motion sickness" and better appreciation of adverse physiologic effects of graphics displays due to slight delay (Frank et al., 1988) or mismatch between imperfect representations of the dozen or so environmental cues that convey visual structure and depth (Palmer, 1998). Advances in understanding the role and representations of these visual cues from the environment in dynamic graphical displays should improve the acceptance and measurement characteristics of driving simulator tools (Rizzo et al., 2002; Kemeny and Fanerai, 2003).

\section{ACKNOWLEDGMENTS}

This research was supported by NIA AG 15071 and NIA AG 17717. 


\section{REFERENCES}

Baloh, R.W. (1997). Neurotology of Migraine. Headache, 37(10): 615-621.

Cass, S., Furman, J., Ankerstjerne, K., et al. (1997). Migraine-related vestibulopathy. Annals of Otology, Rhinology, \& Laryngology, 106(3): 182-189.

Dingus, T.A., Antin, J.F., Hulse, M.C., Wierwille, W.W. (1989). Attentional demand requirements of an automobile moving-map navigation system. Transportation Res, 4, 301315.

Fattal, D., Rizzo, M. (In press). Vestibular system and balance. Ch. 16. In: Rizzo, M., Eslinger, P.J., eds. Principles and Practice of Behavioral Neurology and Neuropsychology. Philadelphia: WB Saunders.

Frank, L.H., Casali, J.G., Wierwille, W.W. (1988). Effects of visual display and motion system delays on operator performance and uneasiness in a driving simulator. Human Factors, 30(2): 201-217.

Hibbard, J.H., Pope, C.R. (1983). Gender roles, illness orientation and use of medical services. Soc. Sci. Med., 17, 129-137.

Kellogg, R.S., Kennedy, R.S., Graybiel, A. (1965). Motion sickness symptomatology of labyrinthine defective and normal subjects during zero gravity maneuvers. Aerospace Medicine, 36, 315-318.

Kemeny, A., Fanerai, F. (2003). Evaluating perception in driving simulation experiments. Trends in Cognitive Sciences 7(1), 31-37.

Kennedy R.S., Lane N.E., Berbaum K.S., Lilienthal M.G. (1993). Simulator sickness questionnaire: an enhanced method for quantifying simulator sickness. International Journal of Aviation Psychology, 3(3): 203-220.

Kennedy, R.S., Lanham, D.S., Massey, C.J., et al. (1995). Gender differences in simulator sickness incidence: implications for military virtual reality systems. $S A F E J, 25,69-76$.

Palmer, S.E. (1999). Vision science: Photons to phenomenology. Cambridge, MA: MIT Press.

Park, A.H., Hu, S. (1999). Gender differences in motion sickness history and susceptibility to optokinetic rotation-induced motion sickness. Aviation, Space, and Environmental Medicine, 70(11), 1077-1080.

Rizzo, M. (In press). Safe and unsafe driving. Ch. 9. In: Rizzo, M., Eslinger, P.J., eds. Principles and Practice of Behavioral Neurology and Neuropsychology. New York: WB Saunders.

Rizzo, M., Jermeland, J., Severson, J. (2002). Instrumented vehicles and driving simulators. Gerontechnology, 1(4), 291-296.

Rizzo, M., Vanderleest, R., Seaba, P., et al. (2003). Synchronous measures of sleepy driver physiology and performance in driving simulator scenarios. 5th International Conference on Fatigue in Transportation: Coping with the 24-Hour Society, Fremantle, Western Australia, March 9 - 13.

Stanney, K.M., Mourant, R.R., Kennedy, R.S. (1998). Human factors issues in virtual environments: A review of the literature. Presence, 7(4), 327-351.

Stanney, K.M., Salvendy, G. (1998). Aftereffects and sense of presence in virtual environments: Formulation of a research and development agenda. International Journal of HumanComputer Interaction, 10(2), 135-187. 
Stanney, K.M., Hash, P. (1998). Locus of user-initiated control in virtual environments: Influences on cybersickness. Presence, 7(5), 447-459.

Thompson, M.K., Brown, J.S. (1980). Feminine roles and variations in women's illness behaviors. Pacific Sociol Rev, 23, 405.

Viirre, E., Bush, D. (2002). Direct effects of virtual environments on users. In K.M. Stanney (Ed.), Handbook of Virtual Environments. Mahwah, NJ: Lawrence Erlbaum Associates, pp. 581-588. 